\title{
Proprioception and Flexibility Profiles of Elite Synchronized Swimmers
}

Nora M.Y. Cho ${ }^{1,2}$, Hedda P. Giorgi ${ }^{1}$, Karen P.Y. Liu ${ }^{3}$, Young-Hyeon Bae ${ }^{4}$,

Louisa M.Y. Chung ${ }^{5}$, Kitchana Kaewkaen ${ }^{6}$, and Shirley S.M. Fong ${ }^{1}$

${ }^{1}$ School of Public Health, University of Hong Kong, Hong Kong

${ }^{2}$ Hong Kong Synchronized Swimming Team (National Squad), Hong Kong

${ }^{3}$ School of Science and Health (Occupational Therapy), Western Sydney

University, Penrith, NSW, Australia

${ }^{4}$ Department of Physical Therapy, CJ Futures Management Institute, Seoul, Republic of Korea

${ }^{5}$ Department of Health and Physical Education, Education University of Hong

Kong, Hong Kong

${ }^{6}$ School of Health Science, Mae Fah Luang University, Chiang Rai, Thailand

Short title: Sensory and flexibility profiles of elite synchronized swimmers

\section{Corresponding author:}

Shirley S.M. Fong

School of Public Health

University of Hong Kong 
301F 3/F HKJCBIR, 5 Sassoon Road

Pokfulam, Hong Kong

Tel: (852)28315260

Fax: (852)28551712

E-mail: smfong@hku.hk 


\begin{abstract}
This study compared the full-body flexibility and joint proprioception (on land and underwater) of (a) 20 elite female synchronized swimmers (mean age \pm standard deviation $=18.5 \pm 1.9$ years) and (b) 20 college female swim team members with no training in synchronized swimming (control participants; (mean age \pm standard deviation $=20.6 \pm 1.3$ years ). Flexibility of the trunk and upper and lower limbs was measured using plastic tape and a goniometer, respectively. Joint proprioception (joint position sense) of the upper and lower limbs on land and underwater was measured by an active joint angle repositioning test. Principle outcome measures were passive joint range of motion (flexibility) and active joint repositioning error (proprioception). Multivariate analysis of covariance revealed that, compared to control swimmers, synchronized swimmers had greater passive joint ranges of motion in the spinal and upper and lower limb joints $(p<0.05)$ and fewer active joint repositioning errors in the shoulder, wrist, and ankle on land $(p<0.05)$ and in the hip and ankle underwater $(p<0.05)$. These results help characterize peak synchronized swimmer capabilities, provide valuable reference details for coaches and may be useful for talent identification, and skill development in this sport.
\end{abstract}


Keywords: Kinanesthesia, anthropometric measures, physical characteristics, peak human performance 


\section{Introduction}

Synchronized swimming combines skills associated with swimming, dancing and gymnastics. Swimmers perform synchronized routines of dance movements in water following musical rhythm. Synchronized swimming has been an Olympic sport since 1984, and it is performed in solo, duet, team, and combination events. Its popularity has increased among young women in recent decades (Sanderson, 2016). The routines practiced by synchronized swimmers involve repeated, complex aquatic gymnastic movements incorporating inverted, supine, and upright positions (Rackham, 1968). Accordingly, these athletes exhibit several sport-specific characteristics, including increased elbow and knee muscle strength, hip adductor flexibility, and artistry (three criteria for evaluation - choreography, musical interpretation and manner of presentation) compared to untrained individuals (Mountjoy, 1999; Yamamura et al., 1999).

Among all the physiological characteristics of synchronized swimmers, flexibility may be most important, because it affects both posture and movement (Li, McClure, \& Pratt, 1996). A previous study confirmed a positive relationship between synchronized swimming performance scores and hip adductor muscle flexibility (Yamamura et al., 1999). Yet, no previous study has explored whether joint-specific flexibilities in other body parts 
distinguish accomplished synchronized swimmers from other experienced sports enthusiasts.

Another important physiological characteristic of synchronized swimmers may be joint proprioception (joint position sense), seen as foundational to coordinated artistic movements (Counil, 2015; Starkes, Gabriele, \& Young, 1989; Sainburg, Poizner, \& Ghez, 1993). Indeed, previous research has reported that highly developed joint position sense was positively associated with expert performance in elite athletes (Muaidi, Nicholson, \& Refshauge, 2009). Joint proprioception is defined as an individual's ability to determine body segment positions and movements in space based on the integration of sensory information from various sensory receptors (Han et al., 2016), including those of the skin, muscle spindles, Golgi tendon organs, and joint (Lundy-Ekman, 2013). Upon submersion in water during synchronized swimming, additional tactile sensory data (i.e., increased skin pressure sensation due to water viscosity) and proprioceptive awareness can assist postural or spatial orientation. The partial immersion of limbs during synchronized swimming might enhance the ability of the central nervous system to analyze differences in skin pressure between submerged and nonsubmerged body parts, and provide useful information for determining verticality or postural orientation (Counil, 2015). Given the neuroplasticity of 
the central nervous system (Lundy-Ekman, 2013), it seems likely that longterm training in synchronized swimming may improve joint proprioception and postural or spatial orientation awareness. Therefore, it is logical to hypothesize that well-trained synchronized swimmers known to exhibit excellent postural control and spatial orientation (Starkes et al., 1989) might have better joint proprioception even than experienced swimmers with no training in synchronized swimming. Additionally, we postulated that joint angle repositioning test may be the best method to assess joint proprioceptive performance (a sport-specific ability) of synchronized swimmers as it explores hemispheric asymmetries in sensorimotor abilities (e.g., postural control) (Han et al., 2016) which are foundational to synchronized swimming performance.

Hence, this study aimed to compare elite synchronized swimmers and experienced college swim team members without training in synchronized swimming with respect to their (a) upper limb, lower limb, and trunk flexibility and (b) joint proprioception (joint angle repositioning) skill on land and underwater. Experienced swimmers who had not undergone training in synchronized swimming (instead of non-swimmers) were recruited as controls for this study because previous research found that swimmers had better joint proprioception than did non-sport-specific healthy research participants (Han, Anson, Waddington, \& Adams, 2014). 


\section{Method}

\section{Participants}

Twenty elite synchronized swimmers were recruited from the Hong Kong National Synchronized Swimming Team via convenience sampling. Twenty healthy age- and sex-matched swimmers, untrained in synchronized swimming, were recruited from the University of Hong Kong Swimming Team. Participants were screened by a trained student researcher using these study inclusion criteria were: (a) aged 18-25; (b) female; (c) > 5 years of experience in synchronized swimming (for synchronized swimming group only) or $>5$ years of experience in swimming generally (for swimming control group); (d) trained in synchronized swimming/swimming $>$ three times per week for two hours per session (for synchronized swimming group only); and (e) demonstrated ability to swim nonstop for 50 meters in a pool. Exclusion criteria were: (a) any history of significant injury that required medical attention in the previous year; (b) any significant musculoskeletal or neurological disorders (e.g., peripheral neuropathy); (c) an open wound or infectious disease (e.g., influenza); (d) current menstruation; or (e) current pregnancy. 
Ethical approval was obtained from the Institutional Review Board of the University of Hong Kong/Hospital Authority Hong Kong West Cluster. Written informed consent was obtained from each eligible participant before data collection. All procedures were performed in accordance with the Declaration of Helsinki.

\section{Outcome measurements}

Data collection was performed by a trained student researcher who was not blinded to the participants' group allocation. It took place at the Kowloon Park and Victoria Park swimming pools in Hong Kong. Demographic data including age, sex, body height, body weight, medical history, and injury history as obtained from each participant via face-to-face interviews. The body

mass index (BMI, in $\mathrm{kg} \cdot \mathrm{m}^{-2}$ ) was subsequently calculated for each participant based on interview data. The participants' synchronized swimming and/or swimming experiences were recorded. Each participant was also invited to complete an International Physical Activity Questionnaire (IPAQ, short form) to document their habitual physical activity (walking, moderate and vigorous physical activities) levels before the physical assessments.

Flexibility 
Upper and lower limb passive joint ranges of motion (PROMs) in the flexion, extension, and abduction directions were measured, using a universal goniometer according to standardized procedures (Clarkson, 2000). The universal goniometer is a valid and reliable tool (ICC: $0.81-0.94)$ for measuring peripheral joint mobility in adults (Gajdosik \& Bohannon, 1987). The detailed procedures for measuring the shoulder, elbow, wrist, hip, knee, and ankle PROMs were described previously by Clarkson (2000). In brief, the proximal body parts (e.g., trunk) were stabilized, and each distal body part (e.g., thigh) moved through the full range of motion in a specific direction. The assessor added an additional force to the distal body part at the end range of motion to evaluate the PROM (flexibility). The goniometer axis was aligned with the tested joint axis, and the movable arm of the goniometer moved together with the distal body part to register the PROM (Clarkson, 2000). A warm-up trial was allowed for all joint PROM measurements to avoid a testing effect (Gajdosik, \& Bohannon, 1987), after which the average PROM value of 3 testing trials per joint movement was documented to improve reliability of the goniometric measurements (Gajdosik, \& Bohannon, 1987). Only the dominant upper limb and lower limb were tested given the differences in PROM between body sides are minimal in healthy young 
females (Macedo, \& Magee, 2008) and the movements of synchronized swimming are largely symmetrical (Rackham, 1968).

The trunk (thoracolumbar spine) flexion, extension, and side flexion PROMs were assessed with the participant in standing and using a cloth measuring tape (marked in $\mathrm{mm}$ ) according to standardized procedures (Clarkson, 2000). This method was found to measure spinal PROMs with satisfactory validity and reliability (Burdett, Brown, \& Fall, 1986). Three trials were conducted and the average distances between the spinous processes of the C7 and S2 vertebrae (trunk flexion), between the jugular notch and the floor (trunk extension), and between the ipsilateral middle finger tip and the floor (trunk side flexion) were calculated and recorded (Clarkson, 2000).

\section{Joint proprioception (on land and underwater)}

The active joint angle repositioning test was used to assess the participants' joint proprioception on land and underwater. The participants were blindfolded and positioned in a lateral recumbent position on their nondominant side next to the swimming pool (i.e., on land). The dominant (tested) limb was supported by the assessor to counterbalance gravity. The tested joint (shoulder, elbow, wrist, hip, knee, or ankle) was first positioned passively in the mid-range of flexion-extension (i.e., starting joint angle). Next, the joint 
was randomly moved by the assessor to a new joint angle (either in the inner or outer range while avoiding extreme flexion/extension) in the sagittal plane. The participant memorized that specific joint angle (3 seconds), and the joint was returned to the starting joint angle. Five seconds later, the participant was asked to actively position the joint to the previous joint angle. The starting and participant-reproduced joint angles were measured using a universal goniometer, and the difference between them was calculated to determine the joint active repositioning error (in degrees), which represented joint proprioception. After a warm up trial, three testing trials were conducted for each joint and used to calculate the mean repositioning error (Fong \& Ng, 2006). Underwater joint proprioception was measured using the same procedures, except that the participant laid on an underwater platform and the tested limb was submerged just below the water surface during the test. This active joint angle repositioning test was used in our previous study (Fong \& Ng, 2006) and was shown to be reliable (ICC: 0.753) on land (Benjeminse, Sell, Abt, House, \& Lephart, 2009).

\section{Statistical Analyses}

Sample size calculation was based on a statistical power of $80 \%$ and an alpha level of 5\% (two-tailed). According to the flexibility and joint 
proprioception results of our pilot trial (not shown), an effect size (Cohen’s d) of 0.92 was assumed in this study. Therefore, a minimum sample size of 20 participants per group was needed to detect significant between-group differences in the flexibility and joint proprioception outcomes. G*Power 3.1.0 (Faul, Erdfelder, Lang, \& Buchner, 2007) was used for the sample size calculation.

SPSS Statistics 20.0 software was used for the statistical analysis. Descriptive statistics were generated to describe all demographic and outcome variables. Data normality was checked using Kolmogorov-Smirnov tests and histograms. The demographic data of the synchronized swimming and control groups were compared using an independent t test. Then, three separate multivariate analyses of covariance (MANCOVAs) were used for inter-group comparisons of (1) whole-body flexibility outcomes, (2) upper limb and lower limb joint proprioception on land, and (3) upper limb and lower limb joint proprioception underwater. The results from multivariate analyses showed the overall effects of group on the outcome variables as well as the corresponding Bonferroni-adjusted P values, thus avoiding the inflation of type I errors association with multiple comparisons. Effect size (partial eta-squared) was also calculated for each outcome variable. By convention, partial eta-squared values of $0.01,0.06$ and 0.14 represent small, medium and large effect sizes, 
respectively (Portney \& Watkins, 2009). A two-tailed significance level of 0.05 was adopted for all statistical tests.

\section{Results}

Twenty elite female synchronized swimmers and 20 female swim team members with no training in synchronized swimming (see above for inclusion/exclusion criteria) were recruited to join the synchronized swimming and control groups, respectively. No participants met any exclusion criteria requiring exclusion from the study following their inclusion criteria screening. All demographic data, habitual physical activity levels, synchronized swimming experience, and general swimming experience are presented in Table 1. Because the ages and physical activity levels differed between the two groups, these two variables were treated as covariates in subsequent multivariate analyses.

\section{[Insert Table 1 about here]}

MANCOVA results revealed that all flexibility outcomes (i.e., upper limb, lower limb, and trunk joint PROMs) were significantly higher in the synchronized swimming group than in the control group (Hotelling's trace = 19.614; $\left.\mathrm{F}_{16,21}=25.743 ; p<0.001\right)$. The mean differences ranged from 1.55 
$\mathrm{cm}$ to $15.15 \mathrm{~cm}$ for trunk flexibility outcomes and from $3.47^{\circ}$ to $32.65^{\circ}$ for extremity PROMs (Table 2).

[Insert Table 2 about here.]

For the joint proprioception (active joint repositioning error) tests on land, the synchronized swimming group generally attained lower error scores than did the control group (Hotelling's trace $=0.945 ; \mathrm{F}_{6,31}=4.880 ; p=0.001$ ). Specifically, the synchronized swimmers exhibited lower error scores at the shoulder (42.6\% less error, $p=0.035)$, wrist (56.5\% less error, $p<0.001)$, and ankle joints (49.7\% less error, $p=0.014$ ) relative to the control group, indicating that the former had better shoulder, wrist and ankle joint proprioception. There were no statistically significant between-group differences in elbow, hip and knee joint repositioning errors (Table 2). Regarding underwater joint proprioception (active joint repositioning error), the synchronized swimmers attained lower error scores overall (Hotelling's trace $\left.=1.166 ; \mathrm{F}_{6,31}=6.024 ; p<0.001\right)$, at the hip $(58.2 \%$ less error, $p=0.016)$ and ankle joints (56.0\% less error, $p<0.001)$, indicating that better hip and ankle joint proprioception underwater relative to the controls. However, the shoulder, elbow, wrist, and knee joint repositioning errors were similar between the two groups $(p>0.05)$ (Table 2). 


\section{Discussion}

To our knowledge, this is the first study to examine the full-body flexibility profiles of elite synchronized swimmers. Per our results, elite synchronized swimmers demonstrated greater trunk, upper limb, and lower limb PROMs (i.e., better full-body flexibility), compared to experienced swimmers with no synchronized swim training. This finding may be explained by two considerations. First, though flexibility is known to be a key performance factor in many sports (Yamamura et al., 1999), and elite synchronized swimmers spend particularly significant amounts of time stretching different body parts on land during weekly training routines; and coaches may apply external forces to swimmers’ extremities (i.e., passive stretching) to achieve still greater joint PROMs (Sands et al., 2008). Therefore, elite synchronized swimmers displayed hypermobile joints (e.g., knee hyperextension) even in comparison to other experienced (non-elite) swimmers who had no synchronized swim training. Second, the buoyancy and turbulence associated with synchronized swimming training could assist movements, thereby further improving joint mobility and flexibility (Association of Swimming Therapy, 1992). Indeed, a comprehensive review article demonstrated that practicing specific movements in water could improve thoracolumbar spine, hip, and shoulder joint mobility (range of 
motion) in both healthy and diseased populations (Geytenbeek, 2002). Of course, these water training advantages might have accrued to both groups of swimmers in our study, and our results suggest that synchronized swimming training may yield even more body flexibility than speed swimming activities. Regarding the joint proprioceptive outcomes, our results revealed that when compared with experienced college swim team members, elite synchronized swimmers demonstrated better upper limb (shoulder and wrist) joint position sense on land, but not underwater. The partial/non-immersion of synchronized swimmers' upper limbs for long periods during training (e.g., eggbeater movement) might explain this differential land and water outcome (Rackham, 1968). The sensory receptors responsible for the accurate spatial positioning/orientation of body parts on land include the muscle spindles, Golgi tendon organs, and joint receptors (Lundy-Ekman, 2013; Reschke et al., 1998), whereas skin receptors and proprioceptive receptors are largely responsible for the accurate positioning of body parts underwater (when normal gravitational sensation is absent) (Counil, 2015). Accordingly, synchronized swim training may have selectively enhanced functioning of the muscle spindles, Golgi tendon organs, joint receptors of the upper limbs and central processing of these sensory signals (Counil, 2015; Han et al., 2016). Hence, the synchronized swimmers had superior upper limb joint 
proprioception on land relative to the control group of college swim team members.

The synchronized swimmers also demonstrated better ankle joint proprioception on land, possibly because, during synchronized swimming, the distal ankle is occasionally partially or not immersed in water (e.g., inverted and supine positions) (Rackham, 1968). Therefore, the sensory receptors responsible for the accurate positioning of body parts on land (muscle spindles, Golgi tendon organs and joint receptors) (Lundy-Ekman, 2013; Reschke et al., 1998) were selectively in their functioning in this population.

In addition, the synchronized swimmers had better underwater ankle joint proprioception than the controls, suggesting that the skin receptors responsible for the accurate spatial positioning of body parts underwater and central processing of these sensory signals (Counil, 2015) were also enhanced through synchronized swimming. Another plausible explanation is that a number of synchronized swimming techniques require forceful ankle plantarflexion against water resistance to maintain body balance (e.g., eggbeater movement) (Homma \& Homma, 2005), which may increase the muscle spindle afferents and, hence, underwater ankle joint proprioception (Dalecki \& Bock, 2013). 
Another novel finding of this study was that the synchronized swimmers had better hip joint proprioception underwater, but not on land, which could be attributed to the large number of underwater kicking techniques practiced by these athletes (Rackham, 1968). For example, during the horizontal kicking and vertical kicking workouts associated with the eggbeater movement, synchronized swimmers’ hip joints are submerged deeply in water (Homma \& Homma, 2005). Accordingly, the hips were surrounded by a high level of water pressure, which could better stimulate the skin receptors around these joints (Counil, 2015). These factors probably enhanced hip joint proprioception underwater. In addition, kicking actively in an aquatic environment could enhance proprioceptive feedback via increases in muscle spindle afferents (Dalecki \& Bock, 2013), which might also mechanistically explain the superior underwater hip joint proprioception observed among elite synchronized swimmers.

In contrast, no significant differences in elbow and knee joint proprioception were observed between the synchronized swimming and control groups, either on land or underwater. We postulated that as both the elbow and knee joints have only one degree of freedom (flexion/extension), these joints might not be very important to synchronized swimming poses (Rackham, 1968). Accordingly, these two joints might have been de- 
emphasized during training. Additional movement analysis studies are needed to confirm our postulation.

Although our findings were generally encouraging, several limitations of this study should be noted when interpreting the results. First, this was a cross-sectional study, and the superior joint flexibility and proprioceptive senses of synchronized swimmers could be attributed to natural ability and the athlete's self-selection to participate in synchronized swimming rather than the result of synchronized swimming training. Further experimental study is needed to establish cause-and-effect relationships between synchronized swimming training, joint flexibility, and proprioception. Second, some data with possible relevance to flexibility and proprioception skills of synchronized swimmers was not explored, including the age of participants at training onset and standardized water temperature measurements (Chow, Yam, Chung, \& Fong, 2017). Third, the validity and reliability of underwater goniometry have not yet been established. Further studies might use an underwater motion analysis system to capture changes in joint angles, rather than a universal goniometer (Rostkowska, 2005). Fourth, swimmers in the control group may participate in water sports that have a high demand on proprioceptive ability (e.g., diving). This could have confounded the results. Fifth, the assessor was not blinded to group allocation and this may have introduced tester bias in the 
assessments. Finally, since the elite synchronized swimmers in our study were young adults, it is not known how well these results might generalize to other age groups or training levels (e.g., recreational synchronized swimmers). Further studies may identify the mechanisms underlying the improved joint flexibility and proprioception we found to be associated with synchronized swimming. A randomized controlled trial may also be conducted to confirm the effects of synchronized swimming on joint flexibility and proprioception in female athletes. Effective training strategies could then be developed to improve sport performance. Nevertheless, our results provided reference values and should benefit the selection and training of peak performing synchronized swimmers. 


\section{References}

1. Association of Swimming Therapy. (1992). Swimming for people with disabilities. ( ${ }^{\text {nd }}$ ed.). London, UK: A \& C Black.

2. Benjaminse, A., Sell, T.C., Abt, J.P., House, A.J., \& Lephart, S.M. (2009). Reliability and precision of hip proprioception methods in healthy individuals. Clinical Journal of Sport Medicine, 19, 457-463.

3. Burdett, R.G., Brown, K.E., \& Fall, M.P. (1986). Reliability and validity of four instruments for measuring lumbar spine and pelvic positions. Physical Therapy, 66, 677-684.

4. Chow, G.C.C., Yam, T.T.T., Chung, J.W.Y., \& Fong, S.S.M. (2017). Effects of postexercise ice-water and room-temperature water immersion on the sensory organization of balance control and lower limb proprioception in amateur rugby players: A randomized controlled trial. Medicine, 96, e6146.

5. Clarkson, H.M. (2000). Musculoskeletal assessment: Joint range of motion and manual muscle strength. Philadelphia, US: Lippincott Williams \& Wilkins.

6. Counil, L. (2015). Field dependence and orientation of upside-down posture in water. Perceptual and Motor Skills, 120, 15-24.

7. Dalecki, M., \& Bock, O. (2013). Changed joint position sense and muscle 
activity in simulated weightlessness by water immersion. Aviation, Space, and Environmental Medicine, 84, 110-115.

8. Faul, F., Erdfelder, E., Lang, A.G., \& Buchner, A. (2007). G*Power 3: A flexible statistical power analysis program for the social, behavioral, and biomedical sciences. Behavior Research Methods, 39, 175-191.

9. Fong, S.M., \& Ng, G.Y. (2006). The effects on sensorimotor performance and balance with tai chi training. Archives of Physical Medicine and Rehabilitation, 87, 82-87.

10. Gajdosik, R.L., \& Bohannon, R.W. (1987). Clinical measurement of range of motion. Physical Therapy, 67, 1867-1872.

11. Geytenbeek, J. (2002). Evidence for effective hydrotherapy. Physiotherapy, $88,514-529$.

12. Han, J., Anson, J., Waddington, G., \& Adams, R. (2014). Sport attainment and proprioception. International Journal of Sports Science and Coaching, 9, 159-170.

13. Han, J., Waddington, G., Adams, R., Anson, J., \& Liu, Y. (2016). Assessing proprioception: a critical review of methods. Journal of Sport and Health Sciences, 5, 80-90. 
14. Homma, M., \& Homma, M. (2005). Swimming: Coaching points for the technique of the eggbeater kick in synchronized swimming based on three

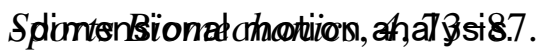

15. Li, Y., McClure, P.W., \& Pratt, N. (1996). The effect of hamstring muscle stretching on standing posture and on lumbar and hip motions during forward bending. Physical Therapy, 76, 836-845.

16. Lundy-Ekman, L. (2007). Neuroscience: Fundamentals for rehabilitation. (3rd ed.). St Louis, Missouri: Saunders Elsevier, 110-112.

17. Macedo, L.G., \& Magee, D.J. (2008). Differences in range of motion between dominant and nondominant sides of upper and lower extremities. Journal of Manipulative and Physiological Therapeutics, 31, 577-582.

18. Mountjoy, M. (1999). The basics of synchronized swimming and its injuries. Clinics in Sports Medicine, 18, 321-336.

19. Muaidi, Q.I., Nicholson, L.L., \& Refshauge, K.M. (2009). Do elite athletes exhibit enhanced proprioceptive acuity, range and strength of knee rotation compared with non-athletes? Scandinavian Journal of Medicine and Science in Sports, 19, 103-112.

20. Portney, L.G., \& Watkins, M.P. (2009). Foundations of clinical research: Applications to practice. (3rd ed.). New Jersey: Pearson Education, Inc. 
21. Rackham, G. (1968). Synchronized swimming. London, UK: Faber and Faber.

22. Reschke, M.F., Bloomberg, J.J., Harm, D.L., Paloski, W.H., Layne, C., \& McDonald, V. (1998). Posture, locomotion, spatial orientation, and motion sickness as a function of space flight. Brain Research Reviews, 28, $102-117$.

23. Rostkowska, E., Habiera, M., \& Antosiak-Cyrak, K. (2005). Angular changes in the elbow joint during underwater movement in synchronized swimmers. Journal of Human Kinetics, 1, 51-66.

24. Sainburg, R.L., Poizner, H., \& Ghez, C. (1993). Loss of proprioception produces deficits in interjoint coordination. Journal of Neurophysiology, $70,2136-2147$.

25. Sanderson, B. (2016). From synchro to decathlon, Olympic playing field still not level for men and women. Retrieved April 10, 2017, from http://www.cbc.ca/news/canada/despite-progress-olympics-still-not-alevel-playing-field-for-men-and-women-1.3663743

26. Sands, W.A., McNeal, J.R., Stone, M.H., Kimmel, W.L., Gregory Haff, G., \& Jemni, M. (2008). The effect of vibration on active and passive range of motion in elite female synchronized swimmers. European Journal of Sport Science, 8, 217-223. 
27. Starkes, J.L., Gabriele, L., \& Young, L. (1989). Performance of the vertical position in synchronized swimming as a function of skill, proprioceptive and visual feedback. Perceptual and Motor Skills, 69, 225-226.

28. Yamamura, C., Zushi, S., Takata, K., Ishiko, T., Matsui, N., \& Kitagawa, K. (1999). Physiological characteristics of well-trained synchronized swimmers in relation to performance scores. International Journal of Sports Medicine, 20, 246-251.

29. Yasuda, T., Nakagawa, T., Inoue, H., Iwamoto, M., \& Inokuchi, A. (1999). The role of the labyrinth, proprioception and plantar mechanosensors in the maintenance of an upright posture. European Archives of Oto-rhinolaryngology, 256, S27-S32. 


\section{Tables}

Table I: Participant characteristics

\begin{tabular}{lccc} 
& $\begin{array}{c}\text { Synchronized } \\
\text { swimming group } \\
(\mathrm{n}=20)\end{array}$ & $\begin{array}{c}\text { Control group } \\
(\mathrm{n}=20)\end{array}$ & $P$ value \\
\hline Age, years & $18.5 \pm 1.9$ & $20.6 \pm 1.3$ & $<0.001$ \\
Sex, $\mathrm{n}$ & 20 females & 20 females & --- \\
Weight, kg & $52.1 \pm 4.0$ & $52.6 \pm 3.9$ & 0.709 \\
Height, m & $1.6 \pm 0.1$ & $1.6 \pm 0.1$ & 0.765 \\
Body mass index, kgm-2 & $20.0 \pm 1.5$ & $20.1 \pm 1.3$ & 0.873 \\
$\begin{array}{l}\text { Physical activity level, } \\
\text { metabolic equivalent }\end{array}$ & & & \\
minutes/week & $5598.8 \pm 414.0$ & $3399.0 \pm 3287.5$ & 0.008 \\
$\begin{array}{l}\text { Synchronized swimming } \\
\text { experience, years }\end{array}$ & $8.5 \pm 2.7$ & & --- \\
$\begin{array}{l}\text { Swimming experience } \\
\text { (synchronized swimming }\end{array}$ & & & \\
experience was included), & & & \\
years & $11.9 \pm 2.0$ & $10.5 \pm 2.5$ & 0.057 \\
\hline
\end{tabular}

Means \pm standard deviations are presented unless otherwise specified. 
Table II: Comparison of the synchronized swimming and control groups

\begin{tabular}{|c|c|c|c|c|}
\hline & $\begin{array}{l}\text { Synchronize } \\
\text { d swimming } \\
\text { group } \\
(\mathrm{n}=20)\end{array}$ & $\begin{array}{c}\text { Control } \\
\text { group } \\
(\mathrm{n}=20)\end{array}$ & $P$ value & Effect size \\
\hline \multicolumn{5}{|c|}{ Joint passive range of motion (flexibility) } \\
\hline & $182.53 \pm$ & $171.90 \pm$ & & \\
\hline Shoulder flexion, degree & 2.91 & 9.05 & $<0.001$ & 0.264 \\
\hline $\begin{array}{l}\text { Shoulder extension, } \\
\text { degree }\end{array}$ & $82.20 \pm 6.86$ & $\begin{array}{c}62.25 \pm 6.43 \\
-2.70 \pm\end{array}$ & 0.001 & 0.550 \\
\hline Elbow extension, degree & $8.80 \pm 4.36$ & 10.48 & $<0.001$ & 0.243 \\
\hline Elbow flexion, degree & $30.10 \pm 6.34$ & $42.08 \pm 5.12$ & 0.002 & 0.410 \\
\hline Wrist flexion, degree & $85.75 \pm 7.04$ & $65.00 \pm 9.31$ & $<0.001$ & 0.556 \\
\hline Wrist extension, degree & $\begin{array}{c}96.93 \pm 7.67 \\
150.80 \pm\end{array}$ & $\begin{array}{c}79.63 \pm 6.29 \\
118.15 \pm\end{array}$ & $<0.001$ & 0.425 \\
\hline Hip flexion, degree & 9.58 & 10.64 & $<0.001$ & 0.745 \\
\hline Hip extension, degree & $\begin{array}{c}37.93 \pm 4.68 \\
62.18 \pm\end{array}$ & $14.38 \pm 5.37$ & $<0.001$ & 0.828 \\
\hline Hip abduction, degree & $\begin{array}{c}10.55 \\
147.45 \pm\end{array}$ & $\begin{array}{c}51.40 \pm 6.91 \\
140.17 \pm\end{array}$ & 0.048 & 0.104 \\
\hline Knee flexion, degree & 7.49 & 9.84 & 0.004 & 0.210 \\
\hline Knee extension, degree & $2.75 \pm 4.00$ & $-4.38 \pm 3.70$ & $<0.001$ & 0.377 \\
\hline $\begin{array}{l}\text { Ankle dorsiflexion, } \\
\text { degree }\end{array}$ & $15.60 \pm 2.89$ & $12.13 \pm 2.54$ & 0.003 & 0.223 \\
\hline $\begin{array}{l}\text { Ankle plantarflexion, } \\
\text { degree }\end{array}$ & $60.73 \pm 4.58$ & $52.13 \pm 5.64$ & $<0.001$ & 0.292 \\
\hline Trunk flexion, cm & $\begin{array}{c}12.60 \pm 1.94 \\
20.20 \pm\end{array}$ & $\begin{array}{c}11.05 \pm 1.71 \\
27.60 \pm\end{array}$ & 0.021 & 0.139 \\
\hline Trunk side flexion, cm & 2.12 & 3.32 & $<0.001$ & 0.509 \\
\hline Trunk extension, cm & $52.50 \pm 2.58$ & $37.35 \pm 4.63$ & $<0.001$ & 0.709 \\
\hline \multicolumn{5}{|c|}{ Joint active repositioning error (on land) } \\
\hline Shoulder, degree & $5.50 \pm 3.76$ & $9.58 \pm 5.03$ & 0.035 & 0.118 \\
\hline Elbow, degree & $6.53 \pm 2.36$ & $9.13 \pm 4.62$ & 0.599 & 0.008 \\
\hline Wrist, degree & $4.93 \pm 3.33$ & $11.33 \pm 4.96$ & $<0.001$ & 0.294 \\
\hline Hip, degree & $5.88 \pm 3.43$ & $8.05 \pm 3.18$ & 0.207 & 0.044 \\
\hline Knee, degree & $6.55 \pm 4.65$ & $11.13 \pm 9.08$ & 0.370 & 0.022 \\
\hline Ankle, degree & $6.43 \pm 5.62$ & $12.78 \pm 8.13$ & 0.014 & 0.156 \\
\hline
\end{tabular}




\begin{tabular}{lcccc}
\hline \multicolumn{4}{l}{ Joint active repositioning error (underwater) } & \\
Shoulder, degree & $5.17 \pm 3.71$ & $9.73 \pm 7.18$ & 0.155 & 0.055 \\
Elbow, degree & $5.13 \pm 5.18$ & $8.50 \pm 7.45$ & 0.263 & 0.035 \\
Wrist, degree & $5.18 \pm 4.84$ & $11.05 \pm 7.16$ & 0.074 & 0.086 \\
Hip, degree & $4.83 \pm 4.85$ & $11.55 \pm 8.77$ & 0.016 & 0.150 \\
Knee, degree & $6.40 \pm 4.37$ & $11.30 \pm 6.74$ & 0.095 & 0.075 \\
Ankle, degree & $5.73 \pm 4.35$ & $13.03 \pm 5.83$ & $<0.001$ & 0.339 \\
\hline
\end{tabular}

Means \pm standard deviations are presented unless otherwise specified. 